\title{
Microwave Assisted Rapid Diagnosis of Plant Virus Diseases by TEM
}

\author{
Bernd Zechmann ${ }^{1}$ and Günther Zellnig ${ }^{2}$
}

1. Baylor University, Center for Microscopy and Imaging, One Bear Place \#97046, Waco, TX 767987046, USA.

2. Karl-Franzens University of Graz, Institute of Plant Sciences, Schuberstrasse 51, 8010 Graz, Austria.

A rapid and unambiguous diagnosis of plant virus diseases is of great importance for agriculture. Investigations of ultrastructural changes induced by viruses within plants by using transmission electron microscopy (TEM) are often necessary to clearly identify the viral agent. Nevertheless, with conventional methods such investigations can take several days and are not suited for the rapid diagnosis of plant virus diseases [1]. Microwave assisted plant sample preparation can help to drastically reduce sample preparation time for TEM investigations with similar or even better ultrastructural results as observed after sample preparation with conventional methods [1,2]. This massive reduction of sample preparation time can be attributed to dielectric heating induced by microwave irradiation which causes a temperature rise inside the whole sample whereas conventional heating starts at the specimen surface. The increase in temperature can then enhance and accelerate the diffusion of reagents, protein crosslinking and the polymerization of the resin [3,4].

In this study microwave assisted sample preparation and negative staining were applied on two model virus-plant systems [Nicotiana tabacum plants infected with Tobacco mosaic virus (TMV), Cucurbita pepo plants infected with Zucchini Yellow mosaic virus (ZYMV)] to clearly diagnose the viral agent and ultrastructural alterations by TEM in less than one day. Sample preparation for ultrastructural and cytohistochemical investigations was performed with a commercially available microwave device. With the help of microwave irradiation sample preparation time was reduced to $136 \mathrm{~min}$ for ultrastructural investigations and to 89 min for cytohistochemical investigations. After cutting and contrasting of the samples ultrastructural investigations revealed typical features of TMV-disease such as virions accumulating in parallel layers within the cytosol of TMV-infected Nicotiana tabacum plants (Figure 1a) and of ZYMV disease like cylindrical inclusions in the cytosol of Cucurbita pepo plants (Figure 1b). Additionally, negative staining of the sap of TMV and ZYMV infected leaves revealed rod shaped viral particles with an average length and width of 280 and $17 \mathrm{~nm}$ for TMV (Figure 1c) and 707 and $12 \mathrm{~nm}$ for ZYMV (Figure 1d). These data were in accordance to the ultrastructural properties and size ranges for these virions described in the literature after samples had been prepared conventionally by chemical fixation and embedding at room temperature. $[5,6,7,8]$. For cytohistochemical investigations sections of TMV-infected leaves were treated with a primary antibody against TMV coat protein and a secondary gold conjugated antibody which took about $100 \mathrm{~min}$. Gold particles bound to TMV coat protein could be clearly identified in less than 4 hours after the beginning of sample preparation in the areas of cells where TMV accumulated in parallel form (Figure 1e).

Summing up, the results presented in this study clearly demonstrate that microwave assisted plant sample preparation for ultrastructural investigtations and cytohistochemical localization of viral coat protein together with negative staining methods enabled a clear diagnosis of plant virus diseases by TEM in less than one day. As these methods could also be applied in the fields of human and veterinary pathology they have a large potential for the rapid diagnosis of diseases in humans and animals. 


\section{References}

[1] B Zechmann and G Zellnig, J. Microsc. 233 (2009), p. 258.

[2] T Kurth et al, Protist 163 (2012), p. 296.

[3] ASY Leong and RT Sormunen, Micron 29 (1998), p. 397.

[4] A De la Hoz et al, Chem. Soc. Rev. 34 (2005), p. 164.

[5] A Gal-On, Mol. Plant Pathol. 8 (2007), p. 139.

[6] ML Smith et al, Virology 348 (2006), p. 475.

[7] AV Reunov et al, Biol. Bulletin 33 (2006), p. 409.

[8] B Zechmann and G Zellnig, J. Virol. Meth. 162 (2009), p. 163.

[9] The authors gratefully acknowledge funding from the Austrian Science Fund (P20619, P22988)

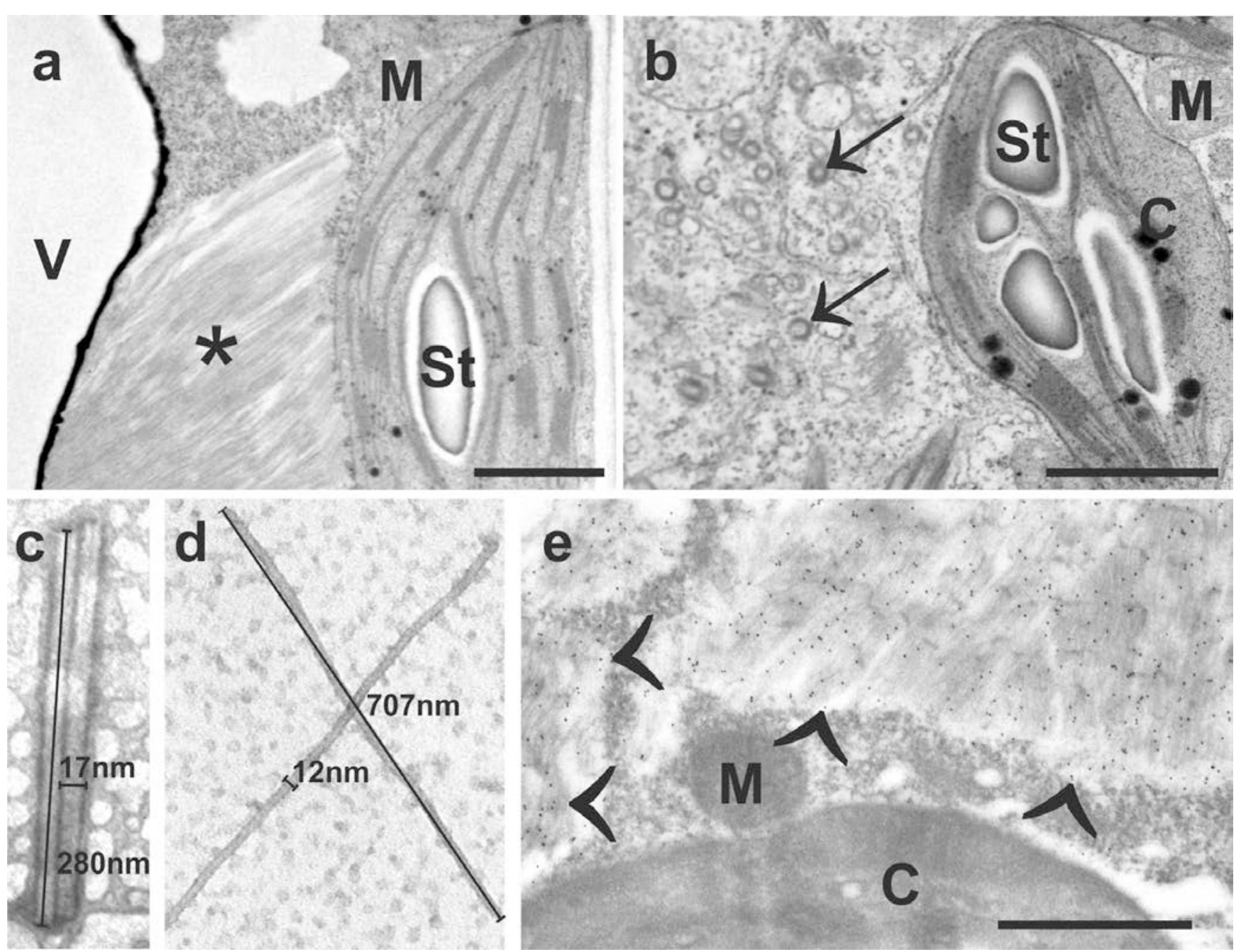

Figure 1. Transmission electron micrographs showing (a) virions (*) in the cytosol of TMV-infected Nicotiana mesophyll leaf cells, (b) cylindrical inclusions appearing as scroll like structures (arrows) in the cytosol of ZYMV-infected Cucurbita mesophyll leaf cells, (c) TMV-particles after negative staining of the sap of TMV-infected Nicotiana leaves, (d) ZYMV-particles after negative staining of the sap of ZYMV-infected Cucurbita leaves, and (e) gold particles bound to TMV coat protein (arrowheads) in the cytosol of TMV-infected Nicotiana leaf cells. $\mathrm{C}=$ chloroplasts with and without starch (St), $\mathrm{M}=$ mitochondria, $\mathrm{V}=$ vacuole. Bars $=1 \mu \mathrm{m}$. 